REPORTES DE TESis DE POSgRado

\title{
Interacción humano computadora: Control de prótesis antropomórfica mediante señales EMG
}

\author{
Karen Cruz, Raymundo Cornejo, Fernando \\ Martínez
}

Publicado: 21 Septiembre 2016

\begin{abstract}
Resumen
El objetivo principal de esta disertación es utilizar un dispositivo que recolecta señales electromiográficas como medio de comunicación entre la actividad muscular del antebrazo y los actuadores de una prótesis mecánica antropomórfica capaz de realizar posiciones de agarre que le permitan al usuario manipular su entorno de una manera natural e intuitiva.
\end{abstract}

Palabras clave: Prótesis; electromiografía; antropomórfico; señales biológicas; wearable device.

\section{Introducción}

En este trabajo se explora una interfaz natural de interacción humano-computadora, por medio de bioseñales, para el control de una prótesis de mano antropomórfica. La información eléctrica de los músculos se puede recolectar, analizar y clasificar de forma tal que los comandos obtenidos permitan desarrollar acciones de control sobre diferentes tipos de interfaces, en este caso una prótesis antropomórfica. Para este tipo de interfaces, resulta importante que exista un desenvolvimiento natural de la prótesis en relación con la intención gesticular proveniente de las señales eléctricas de los músculos que activan una mano biológica. En este trabajo se utiliza una dispositivo EMG disponible en el mercado que permite recolectar señales electromiográficas, se clasifican algunos eventos de agarre de la mano y se controla una prótesis antropomórfica.

\section{Trabajo relacionado}

En relación con la prótesis antropomórfica se deben considerar las posiciones de agarre que se utilizan para manipular el entorno en las actividades cotidianas. Para el desarrollo de su mano prostética Li Jang et al. [1] revisa diferentes patrones de agarre de la mano. Se comparan trabajos que proponen patrones de agarre a partir de la forma de objetos comúnmente manipulados en las actividades cotidianas, se identifican 8 patrones dada las diferentes posiciones que ocupa el dedo pulgar y el resto de los dedos, y aquellos que identifican 16 patrones considerando aspectos geométricos, cinemáticos y dinámicos presentes durante la interacción de la

Cruz, K., Cornejo, R., Martínez, F.

Facultad de Ingeniería, Universidad Autónoma de Chihuahua

Nuevo Circuito Universitario, C.P. 31125, Chihuahua, México

Email: karencc117@gmail.com, rcornejoga@conacyt.mx,

fmartinez@uach.mx mano con su entorno, interrelaciones identificadas por Cutkosky [2] en una estación de una línea de producción. Dentro de todas las actividades diarias que desarrolla un ser humano con la mano los agarres fuertes representan el $35 \%$, (cuando se aprieta el puño), los agarres precisos un $30 \%$, (cuando se combina el pulgar con otros dedos), mientras que los agarres laterales un $20 \%$, (cuando se usan las yemas de los dedos), y otros agarres complejos [3].

El uso de interfaces intuitivas que reconozcan bioseñales del cuerpo humano y que traduzcan estas en comandos de interacción con la computadora no es nuevo. Ashan et al. [4] revisan algunos contextos de aplicación y los retos inherentes con la recolección e interpretación de datos provenientes de dispositivos que leen señales electroencefalográficas, EEG, electrooculográficas, EOG, y electromiogáficas (EMG). Ashan et al. [4] sugieren adicionalmente algunas técnicas de la inteligencia artificial que podrían explorarse para el procesamiento de señales EMG. Para el control específico de una mano robótica Zaho et al. [5] utilizan redes neuronales para clasificar patrones de movimiento individual de los dedos de la mano a partir de un sistema EMG de tres electrodos. Un pequeño resumen de las técnicas revisadas por estos autores se ofrece en la Tabla 1.

Tabla 1. Técnicas de clasificación EMG [5]

\begin{tabular}{|l|l|}
\hline \multicolumn{1}{|c|}{ Algoritmo } & \multicolumn{1}{c|}{ Patrones } \\
\hline $\begin{array}{l}\text { Red neuronal con retropropagación y } \\
\text { red (BPN) and red neuronal tipo } \\
\text { Hopefield }\end{array}$ & $\begin{array}{l}4 \text { movimientos de codo y } \\
\text { muñeca }\end{array}$ \\
\hline Red neuronal con retropropagación & $\begin{array}{l}\text { Movimientos, torque y } \\
\text { ánulo de los 5 dedos }\end{array}$ \\
\hline Red neuronal con retropropagación & $\begin{array}{l}8 \text { tipos de gestos con la } \\
\text { mano }\end{array}$ \\
\hline Red neuronal con lógica difusa & $\begin{array}{l}6 \text { movimientos de mano, } \\
\text { codo y muñeca }\end{array}$ \\
\hline $\begin{array}{l}\text { Red neuronal con retropropagación y } \\
\text { transformada Wavelet }\end{array}$ & $\begin{array}{l}4 \text { movimientos de } \\
\text { antebrazo }\end{array}$ \\
\hline
\end{tabular}

\section{Objetivo de la investigación}

Emplear un dispositivo que proporciona señales electromiográficas como medio de interacción natural humanocomputadora y como recolector de señales electromiográficas, para diseñar un modelo de reconocimiento y clasificación de patrones que proporcione la información necesaria para controlar, 
mediante posturas de agarre, una prótesis mecánica antropomórfica de mano.

\section{Metodología}

\subsection{Recolección de señales}

Obtener, mediante un estándar de recolección de señales, los datos de las señales electromiográficas de 5 sujetos de prueba entre 20 y 50 años de edad que no padecen enfermedades neurodegenerativas, crónicas o de la piel. Los datos recolectados sirven para incursionar en las actividades de acondicionamiento, análisis y clasificación de la señal EMG.

\subsection{Diseño del algoritmo de clasificación}

La metodología de clasificación se basa en el reconocimiento de patrones utilizando modelos que han sido utilizados por diversos investigadores. Se plantea inicialmente trabajar con una red neuronal de retropropagación en virtud de su popularidad de aplicación en estos contextos.

\subsection{Pruebas}

Se realizan pruebas y retroalimentación para verificar la eficiencia del sistema propuesto. Primero, el algoritmo de clasificación de patrones se evalúa con los datos recolectados de 10 sujetos de prueba sanos. Una segunda prueba se realiza en 5 sujetos con amputación parcial de mano, transcubital o desarticulación de muñeca.

\subsection{Implementación}

Se diseña un escenario controlado en el cual los sujetos con amputación manipulan objetos que comúnmente se maniobran al realizar actividades cotidianas.

\section{Resultados actuales}

Hasta el momento se ha trabajado con un prototipo de prótesis con 3 grados de libertad por dedo, de los cuales las articulaciones proximales son activas y las dos distales son pasivas. La comunicación actualmente se está triangulando con una computadora y un microprocesador Arduino como se muestra en la Figura 1.

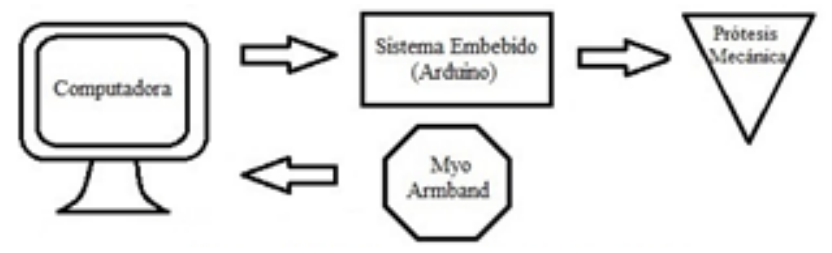

Figura 1. Diagrama de comunicación

Se ha experimentado con los gestos que el brazalete es capaz de reconocer con la programación de fábrica, específicamente con los gestos de mano completamente abierta, puño cerrado y doble palmada. Adicionalmente se ha experimentado con dos agarres que se utilizan con frecuencia en las actividades cotidianas que corresponden a pinza y palmar oblicuo.

\section{Conclusiones potenciales}

El conocimiento adquirido de este estudio provee un esquema para entender los fundamentos de la clasificación de patrones de señales electromiográficas, lo cual se puede emplear como medio de interacción con recursos computacionales. De interés particular es enfocar el trabajo de tesis hacia el desarrollo de experiencias de interacción humano computadora para personas con discapacidad, atendiendo en primera instancia el escenario de una persona que puede estar amputada del brazo.

\section{7 ¿Con qué necesito ayuda?}

Hasta el momento la parte más consumada del proyecto corresponde a la elaboración del algoritmo de clasificación de las señales mioeléctricas en posiciones de agarre que sirven para manipular objetos habituales, por otro lado la parte de las pruebas y la interacción de la prótesis con el usuario no está muy bien definida y existen aún dudas al respecto como ¿Qué implicaciones éticas tiene este estudio en la psicología de los sujetos de prueba? Por ejemplo, en la literatura se establece que muchos pacientes amputados se niegan a utilizar prótesis mecánicas debido a la extensa curva de aprendizaje ¿Cómo se puede lidiar con esta dificultad? De hecho, considerando la etapa de pruebas se anticipa que el uso de la interface EMG solo permitirá sensibilizar a los potenciales usuarios acerca de utilizar su actividad muscular como medio de generación de comandos de interacción HCI. ¿Podrá este nivel de interacción cumplir con las expectativas de los usuarios potenciales?

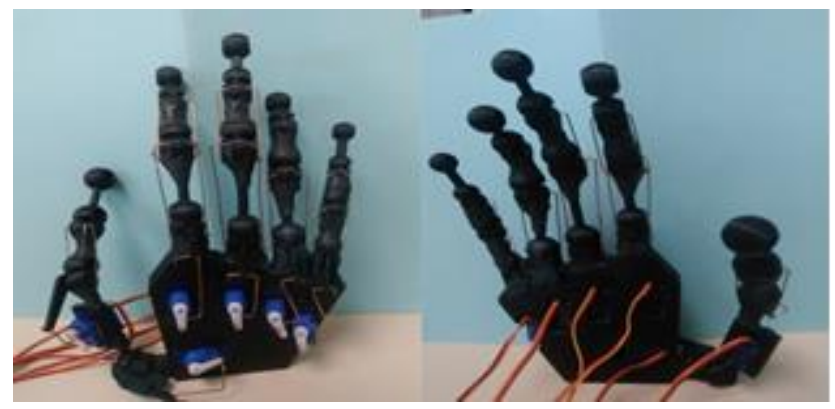

Figura 2. Vista posterior (izquierda) y anterior (derecho) del prototipo

\section{Referencias}

[1] Jiang, L., Zeng, B., Fan, S., Sun, K., Zhang, T., \& Liu, H. (2014, July). A modular multisensory prosthetic hand. In Information and Automation (ICIA), 2014 IEEE International Conference on (pp. 648-653). IEEE.

[2] Cutkosky M. R, De La Rosa S, Dollar A. M. 2011. On Grasp Choice, Grasp Models, and Design of Hands for Manufacturing Tasks. IEEE International Conerence on Robotics and Automation (ICRA). Shanghai, China:41694175

[3] Hoshigawa, S., Jiang, Y., Kato, R., Morishita, S., Nakamura, T., Yabuki, Y., \& Yokoi, H. (2015, August). Structure design for a Two-DoF myoelectric prosthetic hand to realize basic hand functions in ADLs. In 2015 37th Annual International Conference of the IEEE Engineering in Medicine and Biology Society (EMBC) (pp. 4781-4784). IEEE.

[4] Ahsan, M. R., Ibrahimy, M. I., \& Khalifa, O. O. (2009). EMG signal classification for human computer interaction: a review. European Journal of Scientific Research, 33(3), 480501.

[5] Zhao, J., Xie, Z., Jiang, L., Cai, H., Liu, H., \& Hirzinger, G. (2006, February). A five-fingered underactuated prosthetic hand control scheme. In The First IEEE/RAS-EMBS International Conference on Biomedical Robotics and Biomechatronics, 2006. BioRob 2006. (pp. 995-1000). IEEE. 